\title{
Checking unstable properties in distributed testing of communication protocols
}

\author{
Antonio A.F. Loureiro* $\quad$ Samuel T. Chanson ${ }^{\dagger}$ \\ Son T. Vuong ${ }^{\ddagger} \quad$ Osvaldo S.F. de Carvalho*
}

\begin{abstract}
Protocol testing is a run-time activity and we can only hope to detect valid or invalid behaviors in an actual execution of a protocol implementation embedded in a testing environment. In this paper we focus our attention in the validation of dynamic unstable properties during the testing process and afterwards, during normal execution. Dynamic unstable properties define desirable or undesirable temporal evolutions of the behavior of a communication protocol. We shall present a novel on-line distributed algorithm and the corresponding design principles that will improve the testing process and the reliability of a protocol implementation by checking unstable properties in a distributed environment.
\end{abstract}

\section{Keywords}

Unstable properties, distributed protocol testing, distributed algorithms.

\section{INTRODUCTION}

The validation ${ }^{1}$ of global predicates is a fundamental problem in distributed computing that has been used in different contexts such as design, simulation, testing, debugging, and monitoring of distributed programs $[1,7,13,12,15]$. A global predicate may either be stable or unstable. Informally, a stable predicate means that once it becomes true during a computation it will remain true after that, such as a system deadlock. An unstable predicate does not have this

\footnotetext{
*Departamento de Ciência da Computação, Universidade Federal de Minas Gerais, CP 702, 30161-970 Belo Horizonte, MG, Brazil. E-mail: \{loureiro,vado\}@dcc.ufmg.br. Osvaldo Carvalho is on leave at the Institut National des Télécommunications, Evry, France.

† Department of Computer Science, Hong Kong University of Science and Technology, Clear Water Bay, Hong Kong. E-mail: chanson@cs.ust.hk.

Department of Computer Science, University of British Columbia, Vancouver, BC, Canada V6T 1Z4. E-mail: vuong@cs.ubc.ca.

${ }_{1}$ The terms checking, detection, and validation will be used interchangeably in this paper when referring to the validation of desirable behaviors (i.e., predicates or properties) in a protocol.
} 
characteristic. An example is a predicate that relates the length of two queues, each one in a different process. In fact, an unstable predicate may switch from valid to invalid and vice-versa during the execution.

The initial work in the detection of global predicates has concentrated on the validation of stable properties such as distributed termination [5,6] and deadlock detection [2]. Chandy and Lamport [3] proposed an algorithm to take snapshots in a distributed system that became the basis of other algorithms which check stable properties. The word snapshot in this algorithm means the local state of a process $P_{i}$ in the system. Therefore, when $P_{i}$ receives a message to take a snapshot it records its local state and "relays the 'take snapshot' message along all of its outgoing channels" [3]. These 'snapshot' messages are used by a global monitor to construct only consistent global states (see Definition 5 ).

Note that a stable property defined in terms of global predicates can be checked by a global monitor which takes snapshots and constructs the consistent global states. If the stable predicate is found to be true in at least one consistent global state constructed from the snapshots taken in the individual processes then it can be inferred that it will remain in that state at the end of the algorithm. If the predicate is false in the global states constructed, then it was also false at the beginning of the algorithm [3].

Unfortunately this approach does not work for unstable predicates which may be true during an execution but not checked, or found to be true in some states but it may have never happened because the global monitor constructs all possible consistent global states (see [1] for an example of this situation).

The testing process is a run-time activity and we can only hope to detect valid or invalid behaviors in an actual execution of a protocol implementation embedded in a testing environment. In this paper we focus our attention in the validation of dynamic properties during the testing process and afterwards, during normal execution. Dynamic properties define desirable or undesirable temporal evolutions of the behavior of a communication protocol. We shall present a set of novel design principles that will improve the testing process and the reliability of a protocol implementation by checking desirable behaviors. The goals to be accomplished are summarized as follows:

$\triangleright$ Protocol testing:

- Mechanism to check global predicates based on local predicates.

- Identification of consistent global states where any type of predicate can be checked.

- Protocol execution:

- Obtain information to avoid the problem of state build-up.

- Obtain information for use in exception handling.

The rest of this paper is organized as follows. Section 2 describes the formal model used in this paper. Section 3 discusses the tasks involved in the detection of dynamic properties. Section 4 describes the design principles related to the testing process, including the algorithm to detect the properties. Section 5 describes the design principles related to the execution of the protocol implementation. Section 6 discusses the related work. Finally, Section 7 presents the conclusions for this paper.

\section{FORMAL MODEL}

In this section we present some definitions that will be used in the algorithm described in Section 4. Our algorithm is based on the communicating extended finite state machine (CEFSM) model. First we present the nomenclature for identifiers that will be used in this paper. 
Nomenclature 1 (Identifiers) Identifiers can have a subscript and/or a superscript both of which are used to indicate an element in a set. Let $\square_{i}^{x}$ represent an identifier. The subscript $i$ refers to the $i$-th element of $\square$. For example, process $P_{i}$. The superscript refers to the $x$-th element of $\square_{i}$ in the case $\square_{i}$ is also a set.

Definition 2 (Communicating extended finite state machines) A communicating extended finite state machine is a labeled directed graph where vertices represent states and edges represent transitions. A designated vertex represents the initial state of the machine. Transitions are labeled with the pair $\frac{\text { event }}{\text { action }}$. An event is the reception of a message, or an internal event not related to a channel (e.g., a timeout or a service request from a service user). An action is the sending of a message, the starting of a timer, or the assignment of a value to a local variable. The communication between machines is asynchronous. The communicating channels between each pair of machines are not perfect so that messages can be reordered, corrupted or lost.

Formally, a communicating extended finite state machine $P_{i}(i=1 \ldots r)$ is a five-tuple $P_{i}=$ $\left(S_{i}, \Sigma_{i}, \delta_{i}, \lambda_{i}, s_{i}^{0}\right)$, where

- $S_{i}$ is the set of local states of machine $P_{i}$.

- $\Sigma_{i}$ is the set of events and actions. This is represented by the sets $\left\{\Sigma_{\mathcal{E}_{i}}\right\}$ and $\left\{\Sigma_{\mathcal{A}_{i}}\right\}$, respectively. There is also a restriction that machine $P_{i}$ cannot send or receive messages from itself.

- $\delta_{i}$ is the transition function and is defined as $\delta_{i}: S_{i} \times \Sigma_{\mathcal{E}_{i}} \rightarrow S_{i}$.

- $\lambda_{i}$ is the output function and is defined as $\lambda_{i}: S_{i} \times \Sigma_{\mathcal{E}_{i}} \rightarrow \Sigma_{\mathcal{A}_{i}}$.

- $s_{i}^{0}$ is the initial state of machine $P_{i}$.

In the labeled directed graph that represents a CEFSM, a message preceded by a + sign means that it was received, and a message preceded by a - sign indicates that it was sent.

Let $P$ be a protocol specification comprised of processes $P_{1}, P_{2}, \ldots, P_{r}$. Each process is modeled as a CEFSM and they are interconnected by a set of communicating channels $C_{1}, C_{2}, \ldots, C_{s}$. Each process $P_{i}(i=1 \ldots r)$ has a finite set of variables $V_{i}^{1}, V_{i}^{2}, \ldots, V_{i}^{k_{i}}$. In this model, the concept of a global state plays a fundamental role in the correctness of a communication protocol.

Definition 3 (Local state) The local state $\gamma_{i}$ of a process $P_{i}$ is defined as the contents of each local variable $\left(V_{i}^{1}, \ldots, V_{i}^{k_{i}}\right)$ in $P_{i}$.

Definition 4 (Global state) A global state is an $n$-tuple of local states, one for each process $P_{i}$, and is represented as $\Gamma=\left(\gamma_{1}, \ldots, \gamma_{n}\right)$, where $\gamma_{i}$ represents the local state of process $P_{i}$.

In this definition we do not include communication channels since we can encode them as part of each local state.

Definition 5 (Consistent global state) Informally, a global state is consistent if it could occur during an execution and a global clock in the system could be used to label precisely the total order of events. Formally, a global state $\Gamma=\left(\gamma_{1}, \ldots, \gamma_{n}\right)$ is consistent iff for any pair $\left(\gamma_{i}, \gamma_{j}\right) \in \Gamma$, then either $\gamma_{i} \prec \gamma_{j}$ or $\gamma_{j} \prec \gamma_{i}$ for $1 \leq i, j \leq n$ and $i \neq j$, according to the transitive closure of relation $\prec$ between local states.

The set of all consistent global states define exactly the states that could have happened in any computation with respect to the events that occurred in each process. Therefore, predicate values are meaningful only if evaluated in a consistent global state. 
The set of all consistent global states $\Gamma$ define a lattice structure $\mathcal{L}$ and its minimal element is the initial global state $\Gamma^{0}=\left(\gamma_{1}^{0}, \ldots, \gamma_{n}^{0}\right)$. In the lattice $\mathcal{L}$ there is an edge from a node representing a global state $\Gamma^{\alpha}=\left(\gamma_{1}, \ldots, \gamma_{i}^{a}, \ldots, \gamma_{n}\right)$ to a node representing $\Gamma^{\text {succ }(\alpha)}=\left(\gamma_{1}, \ldots, \gamma_{i}^{a+1}, \ldots, \gamma_{n}\right)$ iff there exists an event $e$ that $P_{i}$ can execute in its state $\gamma_{i}^{a}$.

Now we present the definitions of local and global predicates that will be used in Section 4 .

Definition 6 (Local predicate) A local predicate of a process $P_{i}$ is a formula in propositional logic (i.e., a boolean expression) where each term of the formula is a local variable of $P_{i}$. The set of local predicates $\phi$ valid for protocol $P$ can be expressed as:

$$
\phi=\left\{\phi_{1}^{1}, \ldots, \phi_{1}^{p_{1}}, \phi_{2}^{1}, \ldots, \phi_{2}^{p_{2}}, \ldots, \phi_{n}^{1}, \ldots, \phi_{n}^{p_{n}}\right\}=\bigcup_{i=1 . . n} \phi_{i}
$$

Definition 7 (Global predicate) A global predicate of protocol $P$ is a formula in propositional logic (i.e., a boolean expression) where each term of the formula is a local predicate of process $P_{i}$. A set of global predicates $\Phi$ valid for protocol $P$ can be expressed as:

$$
\Phi=\left\{\Phi_{1}, \Phi_{2}, \ldots, \Phi_{t}\right\}
$$

\section{TASKS INVOLVED IN THE DETECTION OF UNSTABLE DYNAMIC PROPERTIES}

The detection of unstable dynamic properties involves two tasks. The first one defines the ways a property (global predicate) can be expressed, and the second is the design of algorithms to detect such properties. Clearly the rules used for expressing the properties will guide the design of algorithms to detect such properties. For instance, a property can be defined in a general way [4] involving relations among variables in different processes. In this case we need to identify all the consistent global states, which can be represented by a lattice, to determine whether the property definitely or possibly occurred [1].

In Section 6 we compare the different solutions proposed in the literature to represent predicates and their algorithms and the representation used in this thesis with the algorithm proposed in Section 4.3.

\section{DESIGN PRINCIPLES RELATED TO TESTING}

This section has four parts. The first part describes how dynamic properties are represented (Section 4.1). The second part explains the basic principles used to detect dynamic properties (Section 4.2). The third part describes in details the algorithm to check dynamic properties based on local predicates (Section 4.3). Finally, the fourth part shows that for a specific type of protocol, namely 3-way handshake protocols, we can compute general properties using this algorithm (Section 4.4).

\subsection{Representation of dynamic properties}

Recall from the discussion in Section 1 that we are interested in checking dynamic properties during the testing phase as well as during normal execution of the protocol after the testing process. In this case, the representation of dynamic properties must consider two important 
requirements. First we need to check properties continuously, and second, a dynamic property should describe a valid trace for the protocol specification.

It is well known from automata theory that the languages accepted by finite automata are the languages defined by regular expressions. Furthermore, regular expressions can define infinite languages which, in our case, represent the valid traces for the protocol specification. From Definition 7 we can see that a global predicate $p$ is a regular expression which defines a finite trace. If we want to extend the global predicate to represent an infinite language, we must include the operation for concatenation of expressions represented by the symbol + as in $p^{+}$.

Definition 8 (Dynamic property) A dynamic property is a global predicate expressed in the form given in Definition 7 which may contain the operation for concatenation of expressions represented by the symbol + .

The way a global predicate is expressed is different from equivalence of a regular expression $L$ and a finite automaton $A_{L}$ in two aspects. First, in the case of language recognition there is only one symbol to be processed at each state of the automaton $A_{L}$. In the case of property detection we shall see that we may have a set of valid local predicates in each state of the automaton $A_{\Phi}$ that corresponds to the property $\Phi$. Second, a dynamic property can only use the symbol + for concatenation of expressions and not the symbol *. (See the discussion following Theorem 10 for not including the symbol *.)

From Definition 8 it follows that a dynamic property can be expressed by a deterministic finite automaton (DFA). In fact an automaton is a convenient representation for properties since:

- it represents the nature of communication protocols, that is, of reactive systems;

- it is compatible with the communicating extended finite state machines model used in this thesis to represent protocols;

- it allows the protocol behavior to be partitioned, that is paths and phases, where paths represent partial traces.

Figure 1-(a) shows a binary relation between local predicates and global predicates.

Let the binary relation $\mu_{i}(i=1 \ldots n)$ from $\phi_{i}$ to $\Sigma$ be defined by

$R_{\mu_{i}}=\left\{\left(\phi_{i}^{x}, \Sigma_{j}\right), \phi_{i}^{x} \in \phi_{i}, \Sigma_{j} \in \Sigma \mid \phi_{i}^{x}\right.$ is a term of $\left.\Phi_{j}\right\}$.

The equivalent matrix representation $M_{\mu_{i}}$ for $R_{\mu_{i}}$ is given in Figure 1-(b). The entry $M_{\mu_{i}}\left[\phi_{i}^{x}, \Sigma_{j}\right]=1$ iff $\phi_{i}^{x}$ is a term in the definition of the global predicate $\Phi_{j}$. Otherwise it is zero.

Note that if column $j$ of $M_{\mu_{i}}$ has all entries equal to zero it means that predicate $\Phi_{j}$ does not contain any local predicate of $\phi_{i}$ and this column can be removed. If line $x$ of $M_{\mu_{i}}$ has all entries equal to zero it means that predicate $\phi_{i}^{x}$ does not appear in any global predicate. The designer should analyze this problem which may be an indication of error.

\subsection{Detection of dynamic properties}

To detect a property we will build the automaton $A_{j}$ that represents the global predicate $\Phi_{j}$. The idea of the detection algorithm is to superimpose onto each process $P_{i}(i=1 \ldots n)$ that implements protocol $P$ a local checking procedure that repeatedly performs the following steps:

(i) check and determine the valid local predicates in the set $\phi_{i}$ when process $P_{i}$ goes to a new state, and move the automaton $A_{j}$ to a new state if there are transitions associated with the valid local predicates; 

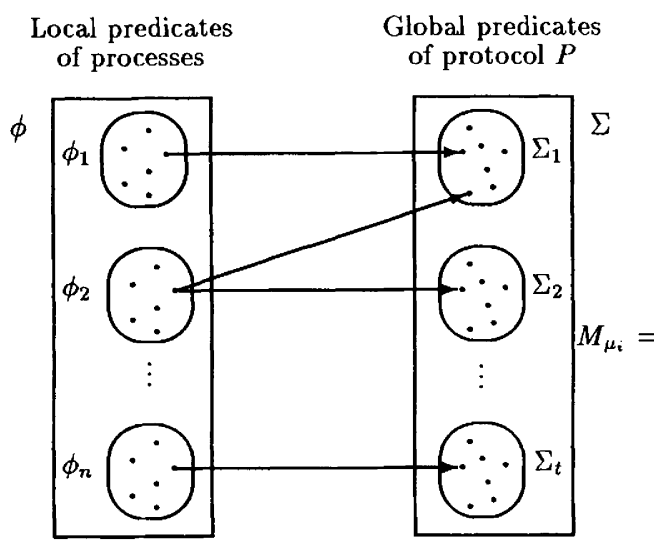

(a) Binary relation $R_{\mu_{i}}$ between local and global predicates.

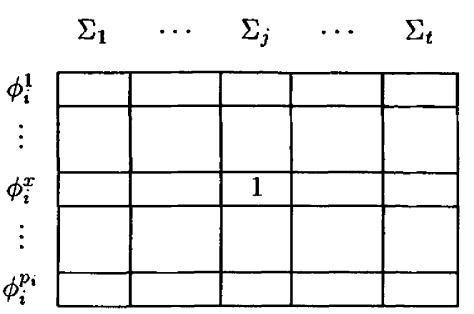

Figure 1 Binary relation $R_{\mu_{i}}$.

(ii) append the current state of the automaton $A_{j}^{i}$ when $P_{i}$ sends a message to another process; and

(iii) check the local predicates as in (i) when $P_{i}$ receives a message from $P_{k}$ and move the automaton $A_{j}$ to a new state based on the current state and the state received from $P_{k}$.

The basic idea of this procedure is to use the automaton $A_{j}$ to keep track of the protocol behavior. If the behavior exhibited by the protocol is valid, the automaton $A_{j}$, which represents property $\Phi$, will eventually reach an accepting state. Of course if the protocol has, for instance, an unspecified reception, the procedure will not be able to check the property since it is superimposed onto each process that implements $P$. Therefore, the protocol should be checked for the common desired properties before applying this algorithm.

Note that this high level algorithm can be applied to any protocol or distributed program in general. In Section 4.3 we shall describe this algorithm in detail. The properties to be checked depend on each protocol and we shall assume that they are part of the input to the algorithm.

In the following we apply this high level algorithm to an example. Suppose we have two processes $P_{1}$ and $P_{2}$ that implement a protocol $P$ such as the ISO Transport Protocol. Furthermore, each process implements a different class of the transport protocol. Given this scenario we would like to make sure, for instance, that the parameters negotiated during the connection establishment remain valid for the entire connection. Let $\phi_{1}=\left\{\phi_{1}^{1}, \phi_{1}^{2}, \phi_{1}^{3}\right\}$ and $\phi_{2}=\left\{\phi_{2}^{1}, \phi_{2}^{2}, \phi_{2}^{3}, \phi_{2}^{4}\right\}$ represent the set of local requirements for processes $P_{1}$ and $P_{2}$ respectively. Let us assume that the property that says that "the parameters selected must remain valid for the entire connection" can be expressed as $\Phi_{j}=\left(\phi_{2}^{1} \vee \phi_{2}^{2}\right)^{+} \wedge \phi_{1}^{1} \wedge \phi_{1}^{2} \wedge\left(\phi_{2}^{4}\right)^{+}$. This predicate can be represented by the automaton in Figure 2-(a). Figure 2-(b) depicts a possible computation for this protocol with the relevant events and messages shown. Next to each event is the set of local predicates that hold at that moment. The current state of the automaton is given inside a circle. Without loss of generality, assume that the protocol $P$ executed the following computation according to the lattice of consistent global states as shown in Figure 2-(c): $(0,0),(0,1),(1,1),(1,2),(2,2),(2,3),(3,3),(4,3),(4,4)$ 
The initial state of the automaton for both processes is $q_{j}^{0}$. When the event $e_{2}^{1}$ occurs the state of the automaton $A_{j}$ in $P_{2}$ goes to $q_{j}^{1}$ after checking the local predicates. At this point, a message $\left\langle m, q_{j}^{1}\right\rangle$ is sent to $P_{1}$. Process $P_{1}$ receives the message and checks its local predicates. At this moment $P_{1}$ is in state $q_{j}^{0}$ and $P_{2}$ in state $q_{j}^{1}$. Note that the only possible transition in the automaton of $P_{1}$ is $\left(q_{j}^{1}, \phi_{1}^{1}, q_{j}^{2}\right)$ and therefore this transition is executed. If the local predicate $\phi_{1}^{1}$ is not valid when event $e_{1}^{1}$ happens then we must take the automaton to its initial state since we cannot have a valid computation that started at a valid state with an invalid prefix. Then computation proceeds as before. When the event $e_{2}^{4}$ occurs, the state of the automaton $A_{j}$ in $P_{2}$ goes to $q_{j}^{4}$ after checking the local predicates. At this point, a message $\left\langle m, q_{j}^{3}\right\rangle$ was received from $P_{1}$. Again, the only possible transition in the automaton of $P_{2}$ is $\left(q_{j}^{3}, \phi_{2}^{4}, q_{j}^{4}\right)$ and therefore this transition is executed. When the state $q_{j}^{4}$ is reached, the property $\Phi_{j}$ is true in process $P_{2}$. Process $P_{1}$ will eventually detect this property if the local predicates associated with $\Phi_{j}$ remain true (i.e., $\phi_{1}^{1}$ and $\phi_{1}^{2}$ ) and $P_{2}$ sends a message to $P_{1}$. If the former condition does not hold it just reflects the temporal behavior of the protocol. If the latter condition does not happen it is because the protocol was not designed to send another message to $P_{1}$.

There are two important points that should be noted in the algorithm proposed. First, the properties do not specify interleaved behaviors of the protocol but conditions that should hold with time. This can be seen from the lattice of consistent global states of the distributed computation as shown in Figure 2-(c). All possible interleavings can be obtained from the lattice. Independent of which path of computation occurs, the property will hold iff along the path the local predicates of each process are valid when the checking is performed. Second, the checking procedure does not modify the behavior given by the protocol specification, it simply appends the current state of the automaton to each message sent.

\subsection{Algorithm to check dynamic properties based on local predicates}

The algorithm to check dynamic properties is given in Figure 3 and is divided into several parts as explained below.

\section{Data structures and algorithm}

$\triangleright$ Data structures

The data structures used in the algorithm are described in the following:

- StateOfAutomaton $[1 \ldots t]$ : each entry of this array represents the current state of the Automaton $A_{j}(j \ldots t)$ in the Process $P_{i}$.

- $L P\left[1 \ldots p_{i}\right]$ : the $x$-th entry $\left(x=1 \ldots p_{i}\right)$ of this array indicates whether the local predicate $\phi_{i}^{x}$ is true at the current state of Process $P_{i}$.

The variable $p_{i}$ represents the cardinality of the set $\phi_{i}$ as given in the Definition 6 .

- ValidLP: set of valid local predicates when process $P_{i}$ moves to a new state.

- EndOfTransition: boolean variable that indicates if there is more transition to be executed.

$\triangleright$ Initialization: Lines $1-3$

Initializes each automaton to its initial state that is by definition the state $q_{j}^{0}$.

$\triangle$ Main part: Lines 4-9

This is the main part of the program that repeatedly checks the properties when the process $P_{i}$ goes to a new state or appends the array StateOfAutomaton to $\langle m\rangle$ when a message is sent. 
$\Phi_{j}=\left(\phi_{2}^{1} \vee \phi_{2}^{2}\right)^{+} \wedge \phi_{1}^{1} \wedge \phi_{1}^{2} \wedge\left(\phi_{2}^{4}\right)^{+}$

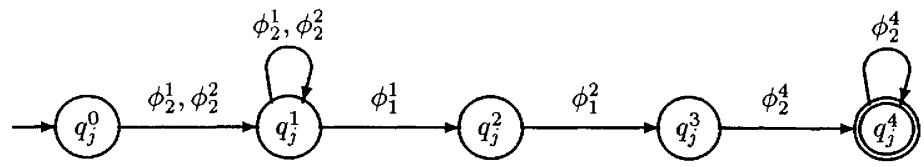

(a) Global property and the corresponding DFA.

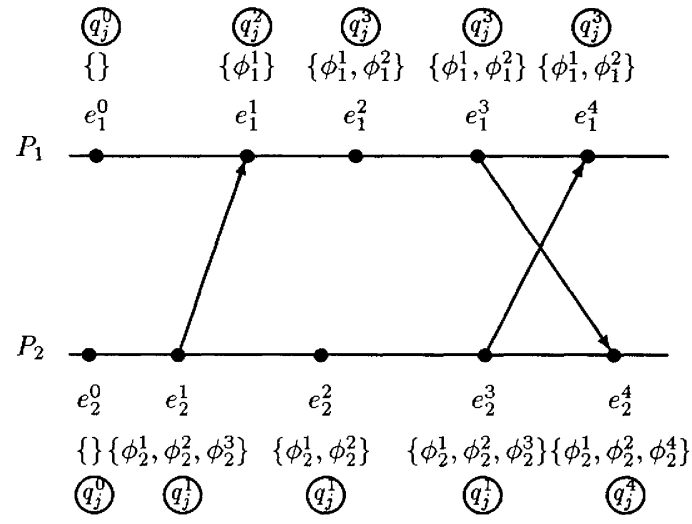

(b) Time-space diagram of the distributed computation with the valid local predicates at each state.

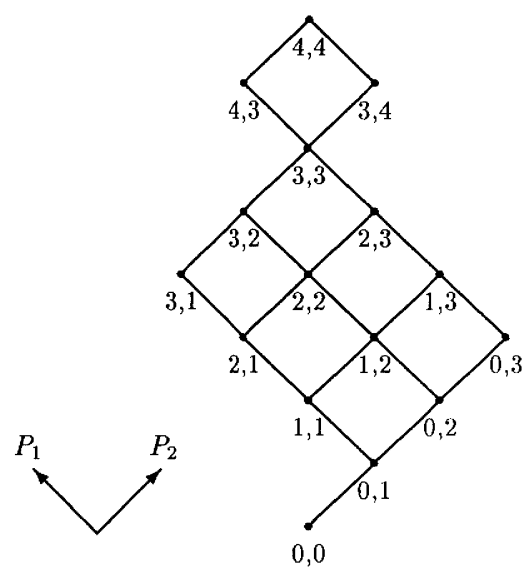

(c) Lattice of consistent global states of the distributed computation.

Figure 2 Checking of properties in a distributed computation.

The part that checks the properties can be seen as a function and is given in lines 10 to 46 .

\section{$\triangleright$ Check each local predicate defined in $P_{i}$ : Lines 10-12}

When process $P_{i}$ goes to a new state, we need to check each local predicate in order to evaluate the global properties. The truth-value of each local predicate depends on its definition. We give a generic function called CheckLP that checks a local predicate.

\section{$\triangleright$ Validation of global properties: Lines 13-44}

In this part we check each property in the set $\Phi$.

$\triangleright$ Determine set of valid local predicates in $\Phi$ at the current moment: Lines 14-23 An automaton $A_{j}^{i}$ can only execute a transition for a local predicate $\phi_{i}^{x}$ iff this local predicate is a term in $\Phi_{j}$ and is valid at the current moment in the process $P_{i}$. If the local predicate is a term of $\Phi_{j}$ but is invalid and there is a transition associated with $\phi_{i}^{x}$ at the current state of $A_{j}^{i}$ then the automaton $A_{j}^{i}$ must be reset. This guarantees the validity of Theorem 9 . 
Theorem 9 An execution (trace) that is validated according to the dynamic property $\Phi$ does not contain an invalid prefix (sequence of invalid local predicates) after its initial state $q_{j}^{0}$.

Proof. By definition, the initial state $q_{j}^{0}$ is a valid state for the automaton $A_{j}$. If $A_{j}$ changes its state from $q_{j}^{0}$ to $q_{j}^{1}$, it is because there is a local predicate $\phi_{i}^{x}$ valid at state $q_{j}^{0}$ and a transition $\left(q_{j}^{0}, \phi_{i}^{x}, q_{j}^{1}\right)$ exists. If the local predicate $\phi_{i}^{x}$ is invalid then it is not possible to execute the transition. Suppose the current state of automaton $A_{j}$ is $q_{j}^{\prime}$ and it is possible to associate a sequence of valid transitions with the path that led the automaton to this state. Now suppose that $\left(q_{j}^{\prime}, \phi_{i}^{x}, q_{j}^{\prime \prime}\right)$ is a possible transition at state $q_{j}^{\prime}$ but the local predicate $\phi_{i}^{x}$ is invalid so that it is not possible to execute this transition at this time. If we leave the automaton at this state it is possible this predicate may become true later on and we will execute this transition. That means that if the property $\Phi$ eventually holds after the state $q_{j}^{\prime}$ later on, $\Phi$ will be true but during the computation there was a false condition. To avoid this inconsistency, we reset the automaton to state $q_{j}^{0}$.

$\triangleright$ Determine next state of the automaton when it receives a message: Lines 24-26 At this point the current state of the automaton $A_{j}$ is given by StateOfAutomaton $[j]$ and a new state $\boldsymbol{q}_{j}^{x}$ is received. Therefore, one of these states has to be chosen so the process of checking the property can be carried on. Before showing how this state is determined, let us examine the situations in which an automaton $A_{j}$ can and cannot move.

The automaton in $P_{i}$ can move if there is a transition $\mathcal{T}=\left(q_{j}^{\prime}, \phi_{i}^{x}, q_{j}^{\prime \prime}\right)$ where $q_{j}^{\prime}$ is the current state of $A_{j}^{i}$ and $\phi_{i}^{x} \in \operatorname{ValidLP}$ (case i). The automaton cannot move when there is a transition $\mathcal{T}$ but $\phi_{i}^{x} \notin \operatorname{ValidLP}$ (case ii), and when there is no transition $\mathcal{T}$ where $\phi_{i}^{x} \in \phi_{i}$ (case iii). If case (ii) occurs it means that the automaton must be reset as shown in Theorem 9 . In case (iii), the automaton can only move from this state if there is a predicate $\phi_{k}^{x}$ in process $P_{k}(k=1 \ldots n)$ that satisfies condition (i). The automaton must move until one of the conditions (ii) or (iii) happens.

Recall from Definition 8 that a global property may contain concatenation of expressions represented by the symbol + . Therefore state $q_{j}^{0}$ has the following characteristics: (a) it cannot be an accepting state for the automaton; (b) there is no transition that ends at $q_{j}^{0}$; and (c) it has just one transition that starts at $q_{j}^{0}$. Condition (a) follows from (b) but it was intentionally given to emphasize the characteristic of this state.

To determine the state of the automaton we need to enumerate the possible combinations in which processes $P_{1}$ and $P_{2}$ stopped moving (i.e., conditions (ii) or (iii) above). The combinations are:

\begin{tabular}{|c|c|c|c|c|}
\hline Combination & 1 & 2 & 3 & 4 \\
\hline$P_{1}$ & (ii) & (ii) & (iii) & (iii) \\
\hline$P_{2}$ & (ii) & (iii) & (ii) & (iii) \\
\hline
\end{tabular}

Condition (ii) means a reset and condition (iii) means that the move depends on the other process.

Suppose that $P_{1}$ is the process that receives a message. (For $P_{2}$ is equivalent.) The first combination is trivial and the state of the automaton $A_{j}^{1}$ remains unchanged. The fourth combination shows that the next state of $A_{j}^{1}$ must be the state of the automaton $A_{j}^{2}$ received from the process $P_{2}$. The second and third combinations are symmetric. Let us analyze the second combination. The automaton $A_{j}^{1}$ is in the state $q_{j}^{0}$ and the automaton $A_{j}^{2}$ is in the state $q_{j}^{x}$. If the state $q_{j}^{x}$ in $A_{j}^{2}$ can be reached directly from state $q_{j}^{0}$ (i.e., with transitions involving only local predicates in 
$P_{2}$ ) then this situation is similar to the fourth combination and the next state of the automaton must be $q_{j}^{x}$. However, if the state $q_{j}^{x}$ cannot be reached directly from $q_{j}^{0}$, it means that process $P_{1}$ has already contributed to this path, i.e., there is at least one transition with a local predicate of $P_{1}$ before reaching state $q_{j}^{x}$. Since there is no transition that ends at $q_{j}^{0}$ (condition (b) above) it means that the automaton $A_{j}^{1}$ was reset and therefore the next state of $A_{j}^{1}$ must be $q_{j}^{0}$ according to Theorem 9. This analysis also applies to the third combination.

Theorem 10 When a process $P_{i}$ receives a message with the state of the automaton $A_{j}$ in $P_{k}$ the next state of automaton $A_{j}$ in $P_{i}$ can be uniquely determined.

\section{Proof. Given above.}

Note that if we allow the concatenation of expressions represented by the symbol $*$ in the definition of global properties, then the conditions (a), (b), and (c) above do not hold anymore and we cannot apply Theorem 10 directly as stated. This does not seem to be an important restriction since if a local property can happen zero or more times, it is probably meaningful if it has to happen at least once.

\section{$\triangle$ Determine whether the automaton can move: Lines 27-40}

At this point we know the set of valid local predicates and the current state of the automaton $A_{j}$ and would like to determine if the automaton can move to a new state. This must be done following the conditions (i), (ii), and (iii) described above.

$\triangle$ Check whether the current state of the automaton satisfies the global property: Lines 41-43

If the current state of the automaton $A_{j}$ is an accepting state then the property $\Phi$ is valid at the current state of process $P_{i}$.

\section{Complexity of the algorithm}

We provide an analysis of the complexity of the algorithm to detect one property $\Phi_{j}$ in lines 13 to 44 . Let $p_{i}$ be the cardinality of the set of local predicates $\phi_{i}$ and $e$ the cardinality of the set of transition functions $\delta_{j}$. The algorithm has five sequential parts. The first part (lines 10-12) validates each local predicate $\phi_{i}^{x}$. Suppose that each predicate can be checked in constant time. Then the validation of the local predicates can be carried out in time $O\left(p_{i}\right)$. The second part (lines 14-23) determines the set of valid local predicates in $\Phi_{j}$ which is also executed in time $O\left(p_{i}\right)$. The third part (lines 24-26) determines the next state of the automaton when it receives a message. There are four cases to be analyzed and this part is executed in constant time. The fourth part (lines 27-40) moves the automaton $A_{j}^{i}$, if possible. Since this depends on the number of transitions in $A_{j}$ this is bounded by $O(e)$. The fifth part checks if the current state of the automaton satisfies the global property and this can done in constant time. Therefore, the five parts together are bounded by $O\left(p_{i}+e\right)$.

\subsection{Special global states in the computation}

In [1] Babaoğlu and Marzullo show that a property $\Phi$ involving relations among variables in different autonomous ${ }^{2}$ processes (i.e., general predicates) can only be detected by building the lattice of consistent global states and then traversing it to determine whether $\Phi$ definitely or

${ }^{2} \mathrm{~A}$ non-autonomous process can only initiate a communication in response to a message received. 
possibly is true. This problem arises because we are considering asynchronous distributed systems where the processes are autonomous and may execute at different speeds.

Begin of algorithm to check dynamic properties

Input: - Set $A=\left\{A_{1}, A_{2}, \ldots\right\}$ of automata that represents the set of properties $\Phi=$ $\left\{\Phi_{1}, \Phi_{2}, \ldots\right\}$ to be detected by process $P_{i}$.

- Set $\phi_{i}=\left\{\phi_{i}^{1}, \ldots, \phi_{i}^{p_{1}}\right\}$ that represents the local predicates valid for process $P_{i}$.

- Matrix $M_{\mu_{i}}$ that represents the binary relation $R_{\mu_{i}}$.

Output: - Validation of each local predicate in $\phi_{i}$.

- Validation of each dynamic property represented by an automaton in the set $A$.

(1) foreach automaton $A_{j} \in A$ do

(2) StateOfAutomaton $[j] \leftarrow q_{j}^{0}$;

(3) od;

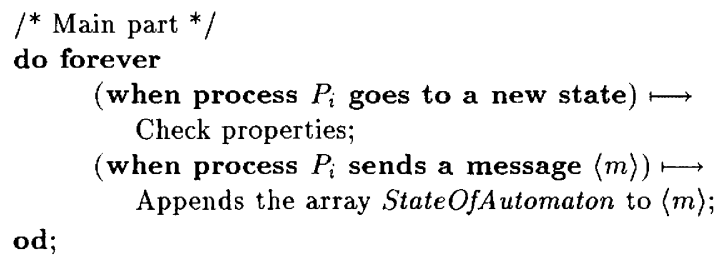

Figure 3 Algorithm to check dynamic properties (Part 1 of 3 ).

In a synchronous distributed system the coordination among processes happens in global synchronization points. Intuitively this means that the computations of the cooperating processes participating in a synchronization converge to a single global state since all computations have to reach that specific point (state). At that synchronization point, general properties can be evaluated. In asynchronous distributed systems there is a similar situation if there is only one autonomous process $P_{A}$ participating in a synchronization and $P_{A}$ is the last process to enter the synchronization state. This is exemplified in Figure 4 with two processes.

Suppose process $P_{2}$ is autonomous and $P_{1}$ is not. To execute a service in this protocol, $P_{2}$ sends a request to $P_{1}$ which may reply with a positive or negative response. If the response is positive, $P_{2}$ can go to a state $\mathcal{S}_{2}$ that represents "service accepted." Note that process $P_{1}$ is already in a state $\mathcal{S}_{1}$ that represents "service accepted" by the time $P_{2}$ receives the response message. Therefore $P_{2}$ was the last process to enter state $\mathcal{S}$ that is our synchronization state. In the executions of Figures $4-(\mathrm{a})$ and 4 -(b) a positive response is represented by events $e_{2}^{2}$, and $e_{2}^{4}$ and $e_{2}^{5}$ respectively. The global states corresponding to these events in the time-space diagrams are in the lattices. This type of protocol is often called 3-way handshake.

Independent of which computation sequence occurred that contains state $\mathcal{S}_{2}$ (e.g., $[\ldots,(3,3),(4,3),(4,4), \ldots]$ or $[\ldots,(3,3),(3,4),(4,4), \ldots]$ in Figure $4-(b))$, we can check general properties at this state since $P_{1}$ is in state $\mathcal{S}$ as well. Furthermore, $P_{1}$ is not autonomous and therefore will remain at $\mathcal{S}_{1}$. The same argument can be extended to systems consisting of two or more processes that are non-autonomous and only one autonomous process. This leads to the following theorem. 


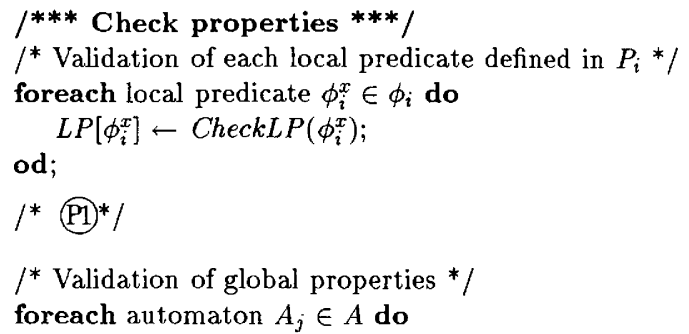

$/$ Determine the set of valid local predicates in $\Phi$ at the current moment. */

(14) $\quad$ ValidLP $\leftarrow\{\}$;

(15) foreach local predicate $\phi_{i}^{x} \in \phi_{i}$ do

(16) if $M_{\mu_{i}}\left[\phi_{i}^{x}, \Sigma_{j}\right]=1$ then

(17) $\quad$ if $L P\left[\phi_{i}^{x}\right]=$ true then

(18) ValidL $P \leftarrow$ ValidLP $\cup\left\{\phi_{i}^{x}\right\}$;

(19) else $/{ }^{*}$ go to the next iteration */

(20) fi;

(21) else /* go to the next iteration */

(22) $\quad$ f;

(23) od;

$/^{*}$ At this point set "ValidLP" contains the local predicates valid at the current moment in $P_{i} .{ }^{*} /$

Figure 3 Algorithm to check dynamic properties (Part 2 of 3).

Theorem 113 -way handshake protocols with two or more non-autonomous processes and only one autonomous process have a global state where general properties can be checked.

Proof. Given above.

\section{DESIGN PRINCIPLES RELATED TO THE EXECUTION}

The algorithm to detect dynamic properties in Figure 3 provides two types of information when process $P_{i}$ goes to a new state:

1. if each local predicate $\phi_{i}^{x} \in \phi_{i}$ is valid or not, and

2. if each global property $\Phi_{j} \in \Phi$ is valid or not.

The first information is provided in point (P1)and the second in point (P2) of the algorithm.

The fault/failure model [10] relates program inputs, faults, data state errors, and failures. A failure is a manifestation of a fault. Although it may or may not occur when there is a fault in the system.

If the protocol implementation runs in "detection mode" (i.e., with the detection algorithm present) we can use these two pieces of information to tackle the problems of state buildup $^{3}$ and exception handling when a fault is detected. Here there is no general solution since

$\overline{3^{3} \text { reactive system such as a communication protocol interacts continuously with its environment. In }}$ 
(44) od;

$/^{*}$ Determine the next state of the automaton if a message $\left\langle m, q_{j}^{x}\right\rangle$ was received from $P_{k}(k=1 \ldots n \wedge k \neq i) .{ }^{*} /$

if received message $\left\langle m, q_{j}^{x}\right\rangle$ then

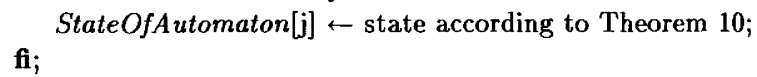

/* Determine whether the automaton can move */

$$
\begin{aligned}
& \text { EndOfTransition } \leftarrow \text { false; } \quad \delta_{j}^{\prime} \leftarrow \delta_{j} ; \\
& \text { while } \neg \text { EndOfTransition do } \\
& \text { if }\left(\exists\left(\text { StateOfAutomaton }[j], \phi_{i}^{x}, q_{j}^{\prime}\right) \in \delta_{j}^{\prime}\right) \\
& \text { if } \phi_{i}^{x} \in \text { ValidLP then } \\
& \text { if StateOfAutomaton }[\mathrm{j}]=q_{j}^{\prime} \text { then } \\
& \qquad \delta_{j}^{\prime} \leftarrow \delta_{j}^{\prime}-\left(q_{j}^{\prime}, \phi_{i}^{x}, q_{j}^{\prime}\right) ;
\end{aligned}
$$

fi;

StateOfAutomaton $[\mathrm{j}] \leftarrow q_{j}^{\prime}$;

else StateOfAutomaton $[j] \leftarrow q_{j}^{0}$;

EndOfTransition $\leftarrow$ true;

fi;

else EndOfTransition $\leftarrow$ true;

f;

od;

$/^{*}$ Check whether the current state of the automaton satisfies the global property.

$*$ I

if StateOfAutomaton $[j] \in Q F_{j}$ then

$/^{*}$ Global predicate $\Phi_{j}$ is valid at this state. ${ }^{*} /$

$l^{*}(\mathrm{P} 2)^{*} /$

fi;

End of algorithm to check dynamic properties

Figure 3 Algorithm to check dynamic properties (Part 3 of 3 ).

these mechanisms depend on the semantics of each protocol. A possible strategy in the case of exception handling is to abort or reinitialize the implementation when "critical" local predicates and properties are not satisfied. This solution is better than allowing an erroneous behavior of the protocol implementation if no action is taken [11]. From the point of view of the other

this case valid input events may lead to an erroneous state in the protocol implementation. When the implementation reaches an erroneous state it may either continue to run but producing erroneous output or may crash and stop. An erroneous state was reached in the implementation because the values associated to its local variables and the contents of its communication channel are not correct according to constraints or requirements given in the protocol specification. Note that this behavior can only be identified if test sequences are as long as the length of the faulty path. 

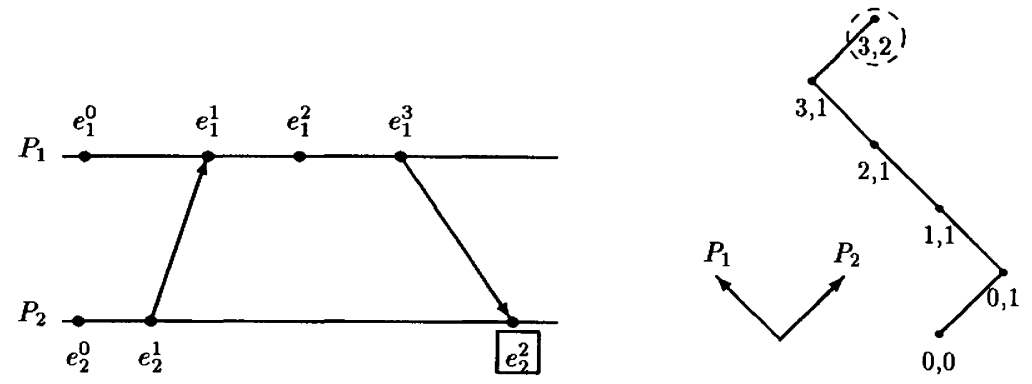

(a) Execution 1 .
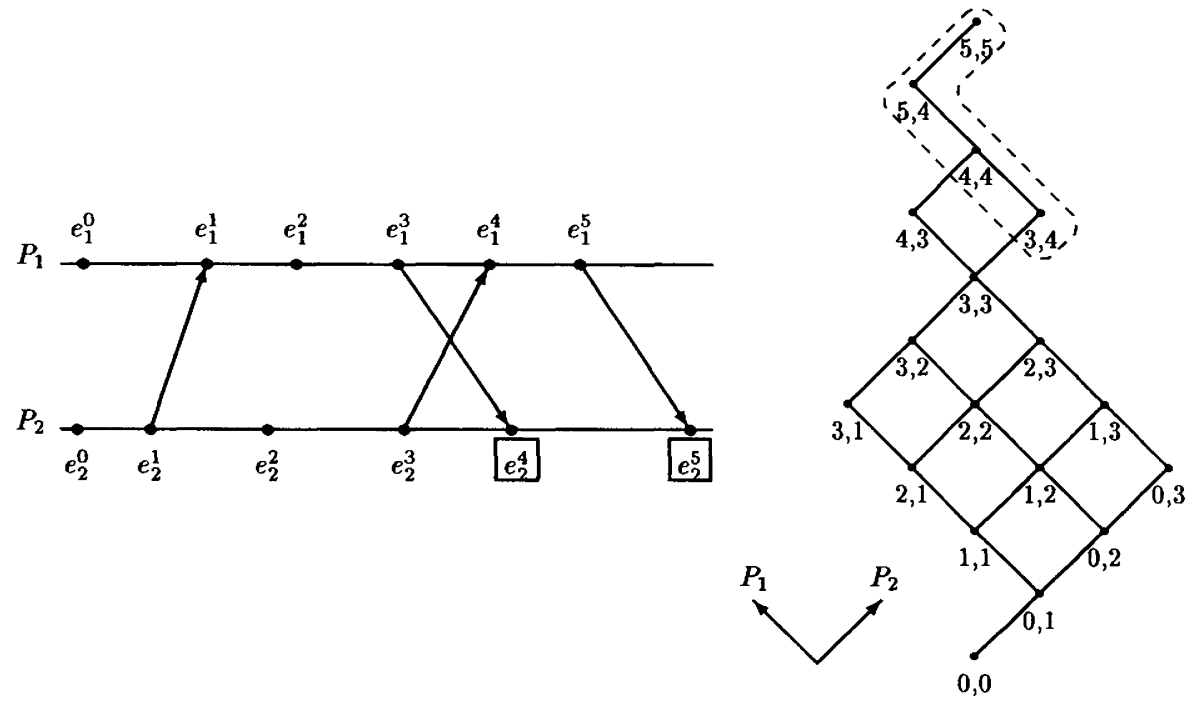

(b) Execution 2 .

Figure 4 Special global states in asynchronous distributed systems.

cooperating processes, the action of aborting or reinitializing an implementation can be seen as a physical failure and therefore the protocol should handle it.

\section{RELATED WORK}

In the following we present the related work in chronological order.

Miller and Choi [9] define linked predicates "that can be ordered by the happened-before relation and are specified by expressions using the $\rightarrow$ operator." They use these predicates in breakpoints in a distributed debugger for halting the system. The halting algorithm is based 
on the Chandy-Lamport snapshot algorithm [3]. Spezialetti and Kearns [14] consider monotonic events that are similar to stable properties and discuss "conditions which must be met in order for specific assertions to be made about an event or the system state." Cooper and Marzullo [4] consider general properties which are intractable in practice since they involve building a lattice of consistent global states that can be exponential in the number of events in the system. Furthermore, all possible paths have to be checked. Hurfin, Plouzeau and Raynal [8] consider unstable nonmonotonic global predicates, called atomic sequences of predicates. These predicates describe global properties by causal composition of local predicates augmented with atomicity constraints that specify forbidden properties. Garg and Waldecker $[7,16]$ define properties in terms of boolean expressions using logic operators. The algorithm to detect these properties is implemented using a global monitor that collects information from all processes and evaluates the predicates. Venkatesan and Dathan [15] also define properties in terms of boolean expressions and give a distributed algorithm to detect these properties. However, the evaluation of properties is performed off-line and they assume that executions of the system are reproducible. Furthermore, they only consider FIFO channels.

The algorithm proposed in this paper considers properties expressed as boolean expressions with concatenation of expressions represented by the symbol + . We give a fully distributed detection algorithm that works on-line and does not modify the protocol specification.

In the case of a centralized monitor $P_{\Phi}$, each process $P_{i}(i=1 \ldots n)$ has to send a message to $P_{\Phi}$ so the property can be checked. Furthermore, if we want to use the information provided by the algorithm during normal execution (see Section 5 ) then the execution in process $P_{i}$ has to be delayed. The algorithm proposed in this paper does not have the cost of sending extra messages since it is distributed and does not delay the execution of process $P_{i}$.

For an overview of the concepts related to this paper and some of the approaches proposed in the literature, the interested reader is referred to [13].

\section{$7 \quad$ CONCLUSIONS}

Verification and testing are two complementary techniques and often used during the protocol development cycle.

In the context of protocol testing, most of the properties that characterize valid or invalid behaviors cannot be expressed in terms of stable properties. In fact, these properties are more properly expressed in terms of desirable or undesirable temporal evolutions of the communication protocol behavior. These properties are inherently unstable since there is no guarantee that they will remain either valid or invalid in a protocol computation.

In this paper we have presented a new algorithm to detect dynamic unstable properties that can be used in the testing of distributed processes (modules) of a communication protocol. This algorithm provides two types of information that can be used for tackling two problems during program execution: state build-up and exception handling.

The algorithm is based on the observation that given a protocol specification there are multiple valid computations (traces) each of which can be defined by a causal precedence order. Dynamic properties are specified by stating conditions (using local predicates) that should hold on all possible computations.

We have also presented a new theorem that can be used to check general properties for a specific type of communication protocol, namely 3 -way handshake protocols.

From the point of view of testing communication protocols or concurrent reactive systems the algorithm presented in this paper is an improvement over the ones presented in $[7,16]$ and [15]. 


\section{REFERENCES}

[1] O. Babaoğlu and K. Marzullo. Consistent global states of distributed systems: fundamental concepts and mechanisms. In Sape Mullender, editor, Distributed Systems, chapter 4, pages 55-96, ACM Press and Addison-Wesley, 1993.

[2] K. Mani Chandy, L.M. Haas, and J. Misra. Distributed deadlock detection. ACM Transactions on Computer Systems, 1(2):144-156, May 1983.

[3] K. Mani Chandy and L. Lamport. Distributed snapshots: Determining global states of distributed systems. ACM Transactions on Computer Systems, 3(1):63-75, February 1985.

[4] R. Cooper and K. Marzullo. Consistent detection of global predicates. In Proc. of the ACM/ONR Workshop on Par. and Dist'd Debugging, pages 167-174, Santa Cruz, CA, USA, 20-21 May 1991. Published as ACM SIGPLAN Notices, 26(12), December 1991.

[5] E.W. Dijkstra and C.S. Scholten. Termination detection for diffusing computation. Information Processing Letters, 11:217-219, August 1980.

[6] N. Francez. Distributed termination. ACM Transactions on Programming Languages and Systems, 2(1):42-55, January 1980.

[7] V.K. Garg and B. Waldecker. Detection of weak unstable predicates in distributed programs. IEEE Trans. on Par. and Dist'd Sys., 5(3):299-307, March 1994.

[8] M. Hurfin, N. Plouzeau, and M. Raynal. Detecting atomic sequences of predicates in distributed computations. In Proc. of the ACM/ONR Workshop on Par. and Dist'd Debugging, pages 32-42, San Diego, CA, USA, 17-18 June 1993. Published as SIGPLAN Notices, 28(12), December 1993.

[9] B.P. Miller and J.-D. Choi. Breakpoints and halting in distributed programs. In Proc. of the 8th Int'l Conf. on Dist'd Computing Sys., pages 316-323, San Jose, CA, USA, 13-17 June 1988.

[10] D. Richardson and M. Thomas. The RELAß model of error detection and its application. In Proc. of the ACM SIGSOFT/IEEE 2nd Workshop on Software Testing, Analysis, and Verification, Banff, Alberta, Canada, July 1988.

[11] J. Rushby. Formal Methods and the Certification of Critical Systems. Technical Report CSL-93-7, SRI International, Computer Science Laboratory, Menlo Park, CA, USA, 1993.

[12] B.A. Schroeder. On-line monitoring: A tutorial. Computer, 28(6):72-78, June 1995.

[13] R. Schwarz and F. Mattern. Detecting causal relationships in distributed computations: In search of the holy grail. Distributed Computing, 7(3):149-174, 1994.

[14] M. Spezialetti and J. P. Kearns. A general approach to recognizing event occurrences in distributed computations. In Proc. of the 8th Int'l Conf. on Dist'd Comput. Sys., pages 300307, San Jose, CA, USA, 13-17 June 1988.

[15] S. Venkatesan and Brahma Dathan. Testing and debugging distributed programs using global predicates. IEEE Transactions on Software Engineering, 21(2):163-177, February 1995.

[16] B. Waldecker and V.K. Garg. Detection of strong predicates in distributed programs. In Proc. of the Third IEEE Symp. on Par. and Dist'd, pages 692-699, Dallas, USA, 2-5 December 1991. 